\title{
International Practicum: Not just another overseas jaunt-Supporting learning objectives and the development of graduate attributes
}

\author{
Sara Geale, Tania Beament, Nick Gibson, Amanda Fowler, Melanie Baker, Gilly Smith * \\ School of Nursing and Midwifery, Edith Cowan University, Perth, Australia
}

Received: April 1, 2015

Accepted: August 6, 2015

Online Published: August 24, 2015

DOI: $10.5430 /$ jnep.v5n $11 \mathrm{p} 96$

URL: http://dx.doi.org/10.5430/jnep.v5n11p96

\begin{abstract}
The nursing profession faces ongoing challenges related to the globalization of nursing education, research, and practice. An online review of undergraduate nursing course offerings and opportunities demonstrates that universities strive to broaden their nursing experience as part of the education experience. Universities and schools of nursing work hard to offer international practicum to students and there are financial implications and risks associated with overseas travel. A literature search using Library One which includes CINAHL Plus, OVID, ProQuest Nursing \& Allied Health Source, and PubMed demonstrated limited description in the literature on whether or not international nursing practicum placements achieve the learning objectives designated in an undergraduate nursing unit, as well as whether or not these practicums support the development of graduate attributes for nusing students. This discussion paper will be one of the first to fill this identified gap in the literature as a reflection on four overseas placements for undergraduate nursing students and how the placements met the stated learning objectives and desired graduate attributes. The Nurse Academics involved in the international practicums critically reflected on the activities of the students and determine the placements support the intent of the units and they help student nurses develop a cross-cultural and international outlook which underwrites provision of patient- and family-centred care for a multicultural population such as that of Australia.
\end{abstract}

Key Words: Nursing education, International practicum, Undergraduate program

\section{INTRODUCTION}

Clinical practice is an indispensable element of nursing learning worldwide. ${ }^{[1]}$ Schools of Nursing globally understand the need for practicums that encourage a development of cultural competency in nursing. ${ }^{[2]}$ As modern societies are marked by rapidly changing economic, cultural, technological, and environmental trends, ${ }^{[3]}$ an increasing global interrelationship demands a generation who can engage in effective problem solving and contribute to life at a local, national, and global level. It is incumbent on nursing scholars to prepare future nurses to be able to participate in a world that demands global competence.

The nursing profession faces ongoing challenges related to the globalization of nursing education, research, and practice. ${ }^{[4]}$ This imposes undergraduate nursing schools to provide education that incorporates a global perspective. An online review of undergraduate nursing course offerings and opportunities demonstrates that universities in North Amer-

\footnotetext{
*Correspondence: Gilly Smith; Email: gillysmith@live.com.au; Address: School of Nursing and Midwifery, Edith Cowan University, Perth, Australia.
} 
ica, Europe and Australia and New Zealand offer students the opportunity to take part in an international clinical placement to broaden their nursing experience as part of the education curriculum. $^{[4]}$ Universities and schools of nursing make a huge committment to administer an international practicum. The schools are required to establish relationships with institutions in the receiving country, send academic staff with the students, and accept the additional financial burden this imposes. Kulwicki (2006) ${ }^{[4]}$ suggests international practicums are not without additional risks related to travel including illness, the risk of political unrest developing in the receiving community, and the possibility that friendships and agreements may be stretched by a lack of cultural awareness by the visitors. ${ }^{[2]}$ However, literature supports the continuation of the international practicum with benefits far outweighing the possible risks. ${ }^{[3]}$ This paper will discuss how international practicums in an Australian school of nursing meet the learning objectives of the nursing curriculum and how the programs support the development of graduate attributes required for the newly graduated registered nurse. The learning objectives and graduate attributes will be discussed in relation to international practicums in locations including Philippines, Tanzania, South India and Thailand.

\subsection{Graduate attributes}

Nursing coursework and clinical practicums place weight on achievement of graduate attributes that support patientcentred, evidence-based nursing practice. Barrie $(2004)^{[5]}$ describes the graduate attributes as the skills, knowledge, and abilities that are required of university graduates to achieve successful completion of an undergraduate degree. Graduate attributes have been a requirement of educational bodies by the Australian government since 1998. ${ }^{[6]}$ Graduate attributes important to nursing practice are the ability to communicate, generate ideas, work collaboratively, think critically, and, importantly in our global climate, have an international outlook and be able to respect other cultures and diversity. ${ }^{[5]}$ Student nurses who experience international clinical rotations provide healthcare in other countries and are challenged to communicate across language and cultural gaps to assess needs, develop therapeutic relationships, and deliver competent care.

\subsection{Learning outcomes}

The Australian Qualification Framework (AQF) $(2013)^{[7]}$ provides oversight of education in Australia. The AQF states successful attainment of a Bachelor Degree qualifies students to undertake specific professional work and provides a foundation for further learning. The AQF defines learning outcomes as the ability to apply knowledge and skills to professional work. Learning outcomes support knowledge as demonstrated by what graduates know and understand, and skills as demonstrated by what the graduate can do (AQF, 2013). ${ }^{[7]}$ The primary learning outcome that validates final year nursing practicum from this particular school of nursing includes demonstration of a collaborative approach with other healthcare professionals in the delivery of care to patients, families and their significant others in a variety of healthcare settings, including the community. Additionally, the students must be able to identify appropriate community agencies and how these agencies meet the biological, psychological, social, spiritual and cultural needs of individuals, families and groups within society. They are required to apply principles of assessment, planning, implementation, and evaluation of nursing care to promote optimal independence and wellness in primary healthcare and acute clinical settings, and to recognize the appropriate use of community health resources. Finally, students should display continued proficiency in medication administration and demonstrate increasing expertise in organization of a patient case load during clinical practice and they should also be able to engage in knowledgeable discourse with other healthcare personnel.

\subsection{Methodology}

Qualitative research methods are used to explore issues, understand phenomena, answer questions by examing unstructured data, and describe that which has previously not been described. ${ }^{[8]}$ In keeping with the expectations of their profession, the nurse educators who accompanied students on overseas practicum placements, committed time to critically reflect on the phenomena of facilitating international clinical placements. Engaging in reflective practice requires individuals to identify assumptions and feelings about their practice and then to speculate how these assumptions and feelings may affect practice. "Reflective practice involves thinking about and critically analysing one's actions with the goal of improving one's professional practice". ${ }^{[9]}$ By examining their feelings and assumptions the nurse academics were able to describe how they (individually) felt the risks and expense associated with international placements could be justified using stories from their experience to demonstrate how the students met course learning outcomes on their placements.

Critical reflective practice in the nursing context often includes seeking feedback or discussing one's feelings and assumptions with a professional peer. Personal critical reflections were shared amongst colleagues in a single School of Nursing and Midwifery, all of whom had been on international clinical practicum. Six Nurse Academics (NA) documented their personal critical reflections about their engagement in international clinical practicums. There was no discrete set of criteria required for these reflections, in- 
stead they were initially generated as a personal professional practice unrelated to any research. The concept of reviewing these reflections for possible publication of shared experience occurred after some professional dialogue determined similar themes may be identifiable in the reflections. Approval to undertake thematic analysis of the reflections to determine if there were consistent themes was granted through the University Human Research Ethics Committee (Smith 12462).

All six reflections were reviewed manually to detect patterns and regularities looking for similar words or sentences relating to student achievement against the learning outcomes and graduate attributes prescribed in the undergraduate program. The analysis was conducted initially by one researcher with their findings checked and confirmed by two other researchers. Polit and Beck (2014) ${ }^{[8]}$ describe member checking as an appropriate form of confirming data findings in thematic analysis.

The shared reflections indicated similar reflections about how well the practicums met the course learning outcomes. As this phenomenon had not previously been described in the literature, the decision was made by the group to articulate these findings and to conduct further research based on critical reflective practice.

\section{INTERNATIONAL PRACTICUM PLACE- MENTS}

In the final year of studies, students from an Australian school of nursing and midwifery are invited to apply for an international practicum. The international practicum consists of two weeks of their required clinical practicum in the first semester of their final year of study. In this unit, the students consolidate previous clinical skills knowledge and apply their clinical competencies to the care of patients in clinical and community settings. Participation in care of the patient or client in the community setting focuses on meeting not only the biophysical health needs but also includes psychosocial, spiritual and cultural needs of the patient, their families and the groups within the community in which the client group lives and works.

The students go through an interview process to determine their suitability for international practicum. The interview considers their reasons for applying for the international practicum as opposed to a domestic practicum. The interviewers take into consideration their current knowledge, skills, and attitudes towards travel and other cultures. After the interviews are complete and the students have accepted their placement, the supervisors meet with the students on a regular basis for the months leading up to the practicum to inform of expectations, rules, regulations, and risks of travel. In addition, prior to the visit the students raise money that is put towards a sustainable project in their practicum country to help improve life in a community or communities. The students make the ultimate decision on where the money will go however, the hosting group in each country, or sponsoring body in Australia, are encouraged to make suggestions regarding appropriate use of the funding relative to local needs.

The Australian school of nursing arranges international practicums in several locations including Philippines, Tanzania, South India and Thailand. These experiences will be discussed below with considerations relating to the development of graduate attributes and learning outcomes achieved.

\subsection{Philippines}

Annually, a group of nursing students and a supervisor travel to the Philippines to take part in a medical mission sponsored by a school of nursing in a Manila-based university. The students visit private, public and mental healthcare facilities in Manila with a focus on community care. The students travel with Filipino physicians and Filipino nursing students to rural communities to support public health visits to those communities. The students live in the local communities, eat locally prepared food, and assist with the daily chores.

In the healthcare clinic, the nursing students assess, educate, and administer medications to patients in conjunction with the physician consult. The students work closely with the Filipino students, Filipino physicians, and with local healthcare workers to deliver care, demonstrating a collaborative approach with other health professionals in the management of patients, families and the greater community. Students are able to practice patient assessment in support of the medical team by taking vital signs and basic patient histories for the physicians. The students are challenged to find ways to communicate with people when there is no common language. They are also obliged to recognize and respect cultural differences in the way healthcare is delivered and the way in which people live.

One of their roles is to provide education to the community and to the community healthcare workers. The students are able to choose which education topics they would like to teach to the healthcare workers. During the 2014 international practicum rotation, the students chose hand hygiene, simple wound dressing, control of diabetes, and range of movement exercise that could be used for bed-ridden patients. They also educated community children on hand hygiene and tooth brushing. The creative side of nursing and the need to aim education at the audience level was demon- 
strated when the students sang a tooth brushing song and developed a game with finger painting to teach the children how to wash their hands.

The 2014 students were also able to support health and wellness in primary healthcare settings when they took part in screening for cervical cancer using mild ascetic acid, or Visual Inspection with Acetic acid (VIA) test. The students found studies supporting the efficacy of the test in sub-Sahara Africa and India with specificity and sensitivity rates close to that of the PAP smear they were familiar with in Australia. ${ }^{[10,11]}$

A second group of students from the 2014 cohort reviewed immunization administration and were able to determine the reason why these immunizations were provided in rural communities. They educated the local community in improving health and wellness. The students achieved learning in theory and the application to practice, were able to recognize the appropriate use of community health resources in these rural areas, and provided education for the community. They presented the information to the other Australian nursing student group and the Filipino students and physicians demonstrating critical thinking, engagement of knowledge with the healthcare team, and cultural awareness.

\subsection{Tanzania}

Global Health Alliance Western Australia (GHAWA) facilitates a group of nursing students from five universities within Western Australia (WA) to observe clinical practice within Tanzania on an annual basis. This placement supports inter-professional collaboration between the five universities within their country of origin, and between countries as the students from WA share education experiences with university students from Tanzania. The students are supported by GHAWA and clinical supervisors from each of the WA universities. The interview process for the Tanzanian practicum is the same as those who attend the other international placements. The focus of this two week international community practicum is to allow the students to experience the Tanzanian health service, global health issues and how healthcare providers work to meet healthcare needs of the community with the resources available in this developing country.

A local clinical supervisor guides the nursing students and supervisors through a hospital in Dar es Salaam, Tanzania, which is the largest city in eastern Africa. The students rotate through busy areas of the health facility including the emergency department, general wards, operating theatres and outpatient areas. Orientation to each area is done by the local nursing staff and the students observe care delivered in that environment. The orientation program helps to develop

Published by Sciedu Press teamwork between the universities and between students and local practitioners. The students observe physician rounds in the paediatric and general surgical wards which support the multidisciplinary approach to healthcare. The students also have the opportunity to travel to rural communities and support delivery of healthcare and health education programs within those communities. During these visits the students interact with local community agencies and evaluate the impact of these agencies on the communities in general and on individuals, families and groups within rural communities.

In the 2013 cohort, the students performed patient care interventions in a public hospital which included removing plaster casts and applying partial casts under the supervision of registered nurses. The students applied knowledge of pain management implementing distracting techniques for patients who were in pain but, due to lack of resources, did not receive medications that are in common use in Australia. Clinical issues noted by the students included complications related to care practices for patients with HIV, malaria and child and maternal health related in the main to lack of resources. The following day was spent in a private hospital operating theatre and the students noted the disparities in the care between the private and public healthcare in Tanzania. The students noted differences in asepsis, infection control, provision of oxygen for patients, and pain management practices. Access to Tanzania's private healthcare system is dependent on the ability to pay for the services.

A debriefing session was held at the end of the each day to support the development of a team approach to care, to foster communication between the groups from the five universities, and to allow for discussion of how funds raised before the students left Australia may best be used to support health resources in the local community. The debriefing sessions allowed for communication of concerns regarding differences in care provided in this setting as opposed to what the students are used to in the home setting.

The Tanzania practicum is fundamental in supporting development of graduate attributes including ability to communicate, team work, development of appraisal skills, the ability to generate ideas and support community health education. While this international practicum may be confronting for students, the placement underwrote the intended learning objective and endorsed the development of the necessary attributes for nurses to work within a multicultural community like Australia.

The 2014 Tanzanian cohort communicated with professionals and the local communities in another country despite the language barrier. The inter-professional collaboration with all five universities within one country and between coun- 
tries as the students from Western Australia shared education experiences with the university students from Tanzania could not have been realized in a domestic placement. Working and living in another country distinct from Australia supported the cross-cultural and international outlook determined as fundamental in working in the global health community.

\subsection{Southern India}

Final year nursing students and a supervisor are hosted by International Village Care Ministry (IVCM) for two weeks in Southern India. The intent of the trip is for students to work within local communities supporting the local healthcare providers in the delivery of care in areas that do not have ease of access to metropolitan healthcare centres.

The Indian practicum requires students to work in situations they have not encountered in previous Australian practicums. They are immersed into a different culture with language barriers and limited resources. Working with appropriate community agencies the students support health checks for the residents of the various homes, including students, staff and elderly residents. They take part in mobile health camps that are arranged for villagers in the surrounding areas. In this activity students are encouraged to apply the principles of community nursing in a variety of primary healthcare settings and work within the confines of the available resources. The students are expected to take an active role in providing health promotion activities for local residents and the school children who attended the mobile health camps.

The 2014 nursing students who attended the Southern India practicum were able to deliver a range of healthcare activities conversant with their scope of practice and sensitive to the bio-psychosocial, spiritual and cultural needs of individuals, families and groups within the Southern Indian society. The students' confidence levels were observed by the accompanying academic nurses to increase with their experiences. They became more adept at crossing the language barrier and managing healthcare issues that are rare in Australia. During the practicum the students became more independent, improved their verbal and nonverbal communication skills, and learned the necessity of working as a team.

This international practicum visit provided a student experience that was both valuable and worthy. The students identified healthcare and health education issues, designed simple programs, and formulated action to address those issues. The students demonstrated a collaborative approach with other health professionals in the management of patients, families and significant others while they applied the principles of community nursing in a primary healthcare setting in Southern India. The students were exposed to a foreign culture and traditions and showed they were able to accept and understand differences and act in a culturally sensitive manner. The supervisor noted the students matured over the time of the visit and felt the challenges faced in the international practicum would be a positive influence on their future nursing careers as registered nurses within the multicultural global community.

\subsection{Thailand}

A strong relationship which has been in place for 10 years with the School of Nursing in Thailand generates clinical practicum placements for student nurses. The students are accommodated on a local campus at a hospitality centre allowing them to experience how the local students live while studying for their nursing degree. Each student is partnered with a Thai undergraduate nursing student and they support and mentor each other throughout the placement. The Thailand experience places a strong emphasis on the "culture of caring" evident in the local healthcare settings. The students participate in a number of clinical settings providing community and home-based care for rural people living with chronic disease and long-term disability. Such placements allow students to work collaboratively with healthcare professionals providing services in these facilities to support care for patients and their families. In contrast to the Australian healthcare system, the families of the patients make a noteworthy contribution to inpatient care which supports early discharge of the patient keeping length of stay to a minimum. The experience affords the students with the opportunity to see patient family focused care in the healthcare setting in another culture.

The Australian and Thai students work as a team to help overcome the language barrier and provide competent patient care within their scope of practice. The students take part in the local community activities, and in lectures and discussions supporting cross cultural knowledge and an international outlook. Further activities include visits to a local orphanage, markets and temples, and a self-sustaining community with a small community clinic funded by the Thai government. The students observe innovative activities focused on creating an independent economy for the villagers who made best use of available community health resources.

The 2014 program presented opportunities for student growth clinically, theoretically and socially with students working with community agencies and evaluation into the impact of these groups on the health of that community. They collaborated with the local agencies to provide healthcare within their current scope of practice overcoming language barriers and differences in culture. 


\section{DisCUSSION}

With the number of international migrants worldwide reaching 232 million in 2013, societies are hugely diverse both linguistically and culturally, and healthcare has taken on a global dimension. ${ }^{[12]}$ Schools of nursing must prepare nurses to deliver culturally competent healthcare. International practicums support this requirement and the investment in these practicums is evident in the manner in which they support the development of expected graduate attributes and meet stated learning objectives of nursing studies in public health. Throughout the 2013-2014 placements in the four settings, the NA reflected the students demonstrated an ability to be creative in their communication, generate ideas, work in teams, think critically and there was a notable growth in the appreciation of other cultures and their lifestyles. The students experienced lifestyle and healthcare in a different culture. While the impact of this experience is yet to be fully realized in the context of their future practice in the multicultural health community of Australia, what can be realized is the practicum in an international setting has provided an insight into the needs of a community outside Australia. The concensus from the educators who accompanied students to the international clinical placements was the students were seen to develop and improve in terms of the the ability to communicate across a language and culture barrier, and also to develop critical appraisal skills relating to current practice in the international setting. Furthermore these groups demonstrated the ability to generate ideas for culturally appropriate education and the team work with the interdisciplinary international group was notable. Future studies will examine how well students felt the international clinical practicums met learning outcomes and student attributes.

\section{Conclusion}

This paper discussed four international nursing practicums that took place in four very different cultures from the reflec- tive perspective of the accompanying NA. The objective of the discussion was to determine if international practicums provide value to nursing students' educational experience and provide them with skills they would be able to use in their healthcare career. The paper considered if such international practicums met the predetermined learning objectives and supported expected graduate attributes. While this was considered in this paper, the team of writers will undertake further studies to determine what learning objectives and graduate attributes are supported by students engaging in international clinical practicum placements. Despite the differences in location and in culture, the critical reflection of the NA's concurred the students in all four of the placements were able to meet the graduate attributes and learning outcomes required. All students demonstrated a collaborative approach with other health professionals, used a varying of community health resources and supported local healthcare agencies, demonstrated competency in healthcare and healthcare education at a level expected for final year students, and worked within a cross cultural team to provide healthcare across language and cultural barriers. Most importantly students from all the settings were able to further develop a cross-cultural and international outlook which supports provision of patient- and family-centred care for a multicultural population such as that of Australia. The themes which could be seen within the critical reflections suggest the authors believe providing international clinical placements for students in undergraduate nursing programs is a successful approach by which students are able to meet both learning outcomes and graduate attributes. Future research will seek to engage the perspective of students who attend international practica, as well as collecting other Nurse Academics' critical reflections of their experience for thematic analysis.

\section{CONFLicts OF InTEREST Disclosure}

The authors declare that there is no conflict of interest.

\section{REFERENCES}

[1] Kim CJ, Park JW, Kang SW. Effects of a collaborative clinical practicum on clinical practice ability and teaching effectiveness among nursing students. Journal of Nursing Education and Practice. 2013; 3(12): 143. http://dx.doi.org/10.5430/jnep.v3 n12p143

[2] Jenkins E, Balneaves LG, Lust A. Students and international nursing shock. Can Nurse. 2011; 107(7): 26-30. PMid:22013845

[3] Educating for global competence: Preparing our youth to engage the world: Asia Society. 2011. Available from: http://asiasociety . org/files/book-globalcompetence.pdf

[4] Kulwicki A. Improving global health care through diversity. Journal of Transcultural Nursing. 2006; 17(4): 396-7. PMid:16946123 http://dx.doi.org/10.1177/1043659606291552

[5] Barrie SC. A research-based approach to generic graduate attributes policy. Higher Education Research \& Development. 2004; 23(3): 261 75. http://dx.doi.org/10.1080/0729436042000235391

[6] Willcoxson L, Wynder M, Laing GK. A whole-of-program approach to the development of generic and professional skills in a university accounting program. Accounting Education: an international journal. 2010; 19(1-2): 65-91.

[7] Australian, Qualifications, Framework, Council. Australian Qualifications Framework Council. Australian Qualifications Framework. 2013.

[8] Polit DF, Beck CT. Essentials of nursing research: Appraising evidence for nursing practice. Philadelphia: Lippincott Williams \& 
Wilkins; 2014.

[9] Imel S. Reflective Practice in Adult Education. ERIC Digest No. 122. 1992.

[10] Adetokunbo AO, Kehinde FO, Oluwaseun OO. The understanding and perception of service providers about the community-based cervical screening in Nigeria. Annals of Tropical Medicine and Public
Health. 2013; 6(6): 637. http://dx.doi .org/10.4103/1755-6 783.140237

[11] Southall JR, Markman M, duPont N. Vinegar screening technique reduced cervical cancer mortality by $31 \%$. HEM/ONC Today. 2013; 14(13): $1-5$.

[12] Nations U. Population facts [Fact Sheet]. 2013. Available from: http://www.un.org/en/index.html 